\title{
Association between posterior uveal melanoma and iris freckles, iris naevi, and choroidal naevi
}

\author{
J W Harbour, M A Brantley Jr, H Hollingsworth, M Gordon
}

Br J Ophthalmol 2004;88:36-38

\begin{abstract}
Aim: To investigate the association between posterior uveal melanoma and iris freckles, iris naevi, and choroidal naevi. Methods: Cross sectional study of 65 patients with posterior uveal melanoma and 218 controls. Iris colour, iris freckles, iris naevi, and choroidal naevi were recorded for each eye of each patient.

Results: Iris freckles were present in 40 (61.5\%) patients with melanoma and $135(61.9 \%)$ controls $(p=0.494)$. Iris naevi were present in four (6.2\%) patients with melanoma and nine $(4.1 \%)$ controls $(p=0.955)$. Choroidal naevi were present in $12(18.5 \%)$ patients with melanoma and $38(17.4 \%)$ controls $(p=0.815)$.

Conclusion: This study did not detect an association between posterior uveal melanoma and iris freckles, iris naevi, or choroidal naevi.
\end{abstract}

U veal melanoma is the most common primary cancer of the eye and the most common non-cutaneous site of melanoma. ${ }^{12}$ The presence of cutaneous freckles and naevi have both been shown to be risk factors for cutaneous melanoma, ${ }^{3-5}$ possibly reflecting a common aetiological factor such as genetic predisposition or ultraviolet light exposure. ${ }^{6}$ Associations have also been reported between posterior uveal melanoma and cutaneous freckles and cutaneous naevi. ${ }^{7-10}$

The relation between uveal melanoma and iris naevi and iris freckles remains unclear. In the 1940s, Reese observed that "pigment freckles of the iris" were more common in eyes with uveal melanoma," and Wilder stated that eyes enucleated for melanoma were more likely to have "pigment cell clusters in the iris" than eyes enucleated for trauma. ${ }^{12}$ However, the modern histopathological correlates of these iris lesions is unclear. A more recent study found an association between uveal melanoma and iris freckles and iris naevi. ${ }^{13}$ However, retrospective data and controls from an ocular oncology referral practice may have biased this analysis. Another study found an association between iris naevi and uveal melanoma, ${ }^{8}$ but $9 \%$ of the patients had atypical mole syndrome, which does not represent a typical population with uveal melanoma. Another study found no association between iris naevi and uveal melanoma. ${ }^{14}$

The relation between uveal melanoma and choroidal naevi is also unclear. One study found no association, but this report did not clarify how the lesions were defined or how the data were collected..$^{15}$ Accurate estimates of the prevalence of choroidal naevi are not available. Hale found choroidal naevi in $6.5 \%$ of necropsy eyes, ${ }^{16}$ and Ganley and Comstock found at least one choroidal naevus in $3.1 \%$ of individuals over $30,{ }^{17}$ but they only evaluated lesions posterior to the equator.

We performed a cross sectional study of 65 patients with posterior uveal melanoma and 218 controls to investigate the relation between posterior uveal melanoma and iris freckles, iris naevi, and choroidal naevi.

\section{PATIENTS AND METHODS}

The study included a consecutive series of patients examined by JWH between February 1998 and October 1999 and who met inclusion criteria into the posterior uveal melanoma group or control group. Age, sex, and race were recorded for each patient. The study was conducted in accordance with the institutional human studies committee. All patients with melanoma were examined prospectively, diagnosed, and treated by one physician (JWH). Inclusion criteria were the presence of an elevated choroidal and/or ciliary body tumour with clinical and ultrasonographic features consistent with uveal melanoma, including low to medium internal reflectivity and a thickness of greater than $3 \mathrm{~mm}$ by standardised A-scan ultrasonography (or documented growth if less than $3 \mathrm{~mm}$ ). Exclusion criteria were: (1) clinical or ultrasound characteristics that were atypical for melanoma, or (2) media opacity that precluded adequate examination of the tumour, iris and choroid. The following tumour features were recorded: tumour dimensions, location (superotemporal, superonasal, inferotemporal, or inferonasal, and anterior versus posterior to the equator), orange pigmentation, exudative retinal detachment, and mushroom configuration. The control group consisted of a consecutive series of patients who were referred for general retinal evaluation by JWH. Exclusion criteria were: (1) referral for suspected neoplasm, (2) media opacity that precluded examination of the iris and choroid of both eyes, (3) iris heterochromia, (4) disorders that could alter iris colour (for example, iris neovascularisation), or (5) a pigmented posterior uveal lesion that could represent a melanoma. Since the melanoma group consisted entirely of white patients at least 20 years old, individuals were included in the control group only if they were white and were at least 20 years old.

Iris colour was graded in a binomial fashion as either light (including blue, green, or grey) or dark (brown). Size and number of iris freckles were recorded for each eye. Iris freckles were defined as flat, discrete areas of pigmentation on the iris surface that are at least $1 \mathrm{~mm}$ in largest basal dimension and do not distort the iris stromal architecture. Iris naevi were counted for each eye. An iris naevus was defined as a tan to brown lesion that occupies and distorts the iris stromal architecture. The following features were noted for each iris naevus: pigmentation, basal dimensions, thickness, location, presence of intrinsic tumour vessels, ectropion uveae, seeding, focal cataract, and angle involvement. Choroidal naevi were counted for each eye using slit lamp biomicroscopy and indirect ophthalmoscopy (with scleral depression when necessary). Fundus examination was performed in an equivalent fashion in both the study and control groups. A choroidal naevus was defined as a choroidal lesion that is at least partially pigmented, at least $1.5 \mathrm{~mm}$ in smallest basal dimension, replaces the choroidal architecture, and obscures the choroidal vessels. Lesions were not included if they were thought to be choroidal freckles, which are typically located between choroidal vessels and do not distort 
the choroidal architecture. The following features were noted for each choroidal naevus: basal dimensions, thickness, location, presence of drusen, subretinal fluid, orange pigment, or surrounding retinal pigment epithelial changes. Hypertrophic and hyperplastic lesions of the retinal pigment epithelium were not included.

Independent sample $t$ tests were performed to test whether the mean age and the mean number of iris freckles were the same in the control and melanoma groups. $\chi^{2}$ Analysis was performed to test the association between (1) sex and group membership, (2) iris colour and group membership, (3) prevalence of iris freckles and iris colour, iris naevi, choroidal naevi, or melanoma location, (4) prevalence of iris naevi and iris colour, choroidal naevi, or melanoma location, and (5) prevalence of choroidal naevi and iris colour, melanoma location, or group membership. Statistical significance was asserted if $p<0.05$. Statistical power was determined by calculating the number of patients in each group that would be required to detect a significant difference between groups, assuming a two sided $\mathrm{p}$ value of 0.05 , statistical power of $80 \%$, and constancy of other variables.

\section{RESULTS}

The melanoma group included 65 patients and the control group 218 patients. Table 1 summarises the clinical information. Control patients were diagnosed with macular degeneration (70 patients), diabetic retinopathy (36 patients), posterior vitreous separation (17 patients), retinal vascular occlusion (15 patients), cystoid macular oedema (10 patients), and other miscellaneous retinal conditions (70 patients). There was a non-significant difference in the mean age of the control group (68.4 years; range, 20-95 years) versus the melanoma group (62.6 years; range $24-86$ years). There was a higher proportion of males in the melanoma group $(63.1 \%)$ than in the control group $(41.7 \%)$. None of the patients in either group had a family or personal history of cutaneous dysplastic naevi or cutaneous melanoma. There was a higher proportion of patients with light iris colour in the melanoma group $(83.1 \%)$ than the control group $(72.5 \%)$, but this difference was not statistically significant $(\mathrm{p}=0.084)$. Table 2 summarises statistical analysis.

\section{Iris freckles}

Iris freckles were present in $40(61.5 \%)$ of the melanoma group and $135(61.9 \%)$ of the control group $(p=0.494)$. Mean freckle number per eye was 1.91 for the melanoma

Table 1 Patient and melanoma characteristics

\begin{tabular}{lll}
\hline Patient and tumour characteristics & $\begin{array}{l}\text { Melanoma } \\
\text { group }\end{array}$ & $\begin{array}{l}\text { Control } \\
\text { group }\end{array}$ \\
\hline Number of patients (n) & 65 & 218 \\
Age (mean) & 62.6 & 68.4 \\
Sex (n,\%) & $41(63.1 \%)$ & $91(41.7 \%)$ \\
$\quad$ Male & $24(36.9 \%)$ & $127(58.3 \%)$ \\
$\quad$ Female & 12.9 & - \\
Melanoma size (mean, mm) & 10.2 & - \\
$\quad$ Largest basal dimension & 6.04 & - \\
$\quad$ Smallest basal dimension & $10(15.4 \%)$ & - \\
Thickness & $23(35.4 \%)$ & - \\
Melanoma location by quadrant (n,\%) & $11(16.9 \%)$ & - \\
$\quad$ Superonasal & $21(32.3 \%)$ & - \\
Superotemporal & & \\
Inferonasal & & \\
Inferotemporal & $21(32.3 \%)$ & - \\
Melanoma location relative to equator $(\mathbf{n}, \%)$ & $44(67.7 \%)$ & - \\
$\quad$ Anterior &
\end{tabular}

group and 1.74 for the control group $(p=0.588)$. Iris freckles were more likely to be detected in eyes with light irides $(p<0.001)$. There was a strong association with the number of iris freckles between fellow eyes in both groups $(p=0.006)$. There was a trend towards association between the presence of iris freckles and iris naevi $(p=0.067)$, and there was a significant association between the number of freckles and the presence of iris naevi for the control and melanoma groups combined $(p=0.032)$ and for the control group alone $(p=0.021)$. There was no association between the presence of iris freckles and the presence of choroidal naevi $(p=0.822)$ or melanoma $(p=0.955)$. To detect $a$ statistically significant difference between the melanoma group and control group with $80 \%$ power, approximately 37168 patients per group would be required.

\section{Iris naevi}

The prevalence of iris naevi was $6.2 \%$ in the melanoma group and $4.1 \%$ in the control group $(p=0.494)$. Iris naevi were more likely to be detected in eyes with light irides $(p=0.385)$. There was no association between iris naevi and choroidal naevi $(p=0.822)$ or melanoma location $(p=0.420)$. To detect a statistically significant difference between the melanoma group and control group with $80 \%$ power, approximately 1863 patients per group would be required.

\section{Choroidal naevi}

Choroidal naevi were present in 12 (18.5\%) of the melanoma group and $38(17.4 \%)$ of the control group $(p=0.815)$. There was no association between the presence of choroidal naevi and iris colour $(p=0.385)$ or melanoma location $(p=0.276)$. To detect a statistically significant difference between the melanoma group and control group with $80 \%$ power, approximately 5792 patients per group would be required.

\section{DISCUSSION}

There is a well established relation between cutaneous freckles, naevi, and melanoma, whereas controversy persists surrounding the analogous relation between posterior uveal melanoma and various uveal melanocytic lesions. This study did not identify an association between posterior uveal melanoma and iris naevi, iris freckles, or choroidal naevi. However, we provide new estimates of prevalence for iris naevi and choroidal naevi that may give more accurate information regarding these lesions in the white population.

Eyes with iris freckles were more likely to have an iris naevus, suggesting that these lesions may be aetiologically linked with each other but not with posterior uveal melanoma. However, both iris freckles and iris naevi were more likely to be detected in eyes with light irides, so the apparent association between these iris lesions may simply reflect a greater difficulty detecting these lesions in darkly pigmented irides.

The prevalence of choroidal naevi (18.5\% in the melanoma group and $17.4 \%$ in the control group) was significantly higher than previous pathological and clinical estimates, ${ }^{16}{ }_{17}$ which may be the result of our prospective data collection and use of indirect ophthalmoscopy to inspect the entire posterior uveal tract. However, since we did not obtain pathological confirmation, it is possible that some lesions that were diagnosed as naevi were in fact non-neoplastic collections of pigmented cells, which could lead to an overestimate of the prevalence of naevi. Nevertheless, most of the lesions diagnosed as naevi had other clinical features consistent with that diagnosis, such as drusen and surrounding retinal pigment epithelial atrophy.

We did not find an association between choroidal naevi and posterior uveal melanoma. To our knowledge, this 


\begin{tabular}{|c|c|c|c|c|}
\hline Clinical feature & Binomial score & Control group & Melanoma group & p Value \\
\hline \multirow[t]{2}{*}{ Iris colour } & Light & 158 (72.5\%) & $54(83.1 \%)$ & \\
\hline & Dark & $60(27.5 \%)$ & 11 (16.9\%) & 0.084 \\
\hline \multirow[t]{2}{*}{ Iris freckles } & Absent & $83(38.1 \%)$ & $25(38.5 \%)$ & \\
\hline & Present & 135 (61.9\%) & $40(61.5 \%)$ & 0.955 \\
\hline \multirow[t]{2}{*}{ Iris naevi } & Absent & 209 (95.9\%) & 61 (93.8\%) & \\
\hline & Present & $9(4.1 \%)$ & $4(6.2 \%)$ & 0.955 \\
\hline \multirow[t]{2}{*}{ Choroidal naevi } & Absent & 180 (82.6\%) & 53 (81.5\%) & \\
\hline & Present & 38 (17.4\%) & $12(18.5 \%)$ & 0.815 \\
\hline
\end{tabular}

question previously has not been addressed in the literature, except as a brief mention in a review article. ${ }^{15}$ This negative result is somewhat surprising in light of the strong correlation between cutaneous naevi and melanoma, and the common assumption that many posterior uveal melanomas arise from pre-existing choroidal naevi. ${ }^{3-5} 18$ The higher prevalence for choroidal naevi suggests that fewer choroidal naevi may convert to melanomas than was previously assumed.

An advantage of our study design is the prospective data collection by a single physician using consistent clinical diagnostic criteria. A potential disadvantage is the use of patients referred for retinal evaluation as a control group. However, the control group demographically resembled the melanoma group, and none of the patients in either group had a history of ocular melanocytosis, dysplastic naevus syndrome, or other known predisposition syndromes for uveal melanocytic lesions.

In summary, this study provides the first cross sectional estimates of prevalence for iris freckles, iris naevi, and choroidal naevi in a white population based on prospective data collection. We found no statistical association between posterior uveal melanoma and iris freckles, iris naevi, or choroidal naevi. We conclude that the aetiological links between these posterior uveal melanomas and these benign uveal melanocytic lesions are sufficiently weak as to be undetected by the statistical power of this study. Additional studies are needed to verify these prevalence rates and to further investigate the relation between uveal pigmentation and posterior uveal melanoma.

\section{ACKNOWLEDGMENTS}

The Biostatistics Core Facility is supported by a grant from the National Eye Institute.

\section{Authors' affiliations}

J W Harbour, M A Brantley, M Gordon, Department of Ophthalmology and Visual Sciences, Washington University, St Louis, MO, USA H Hollingsworth, Department of Occupational Therapy, Washington University, St Louis, MO, USA
Correspondence to: J. William Harbour, MD, Department of Ophthalmology and Visual Sciences, Washington University School of Medicine, Box 8069, 660 S Euclid Ávenue, St Louis, MO 63110, USA; harbour@vision.wustl.edu

Accepted for publication 26 May 2003

\section{REFERENCES}

1 Egan KM, Seddon JM, Glynn RJ, et al. Epidemiologic aspects of uveal melanoma. Surv Ophthalmol 1988;32:239-51.

2 Scotto J, Fraumeni JF Jr, Lee JA. Melanomas of the eye and other noncutaneous sites: epidemiologic aspects. J Natl Cancer Inst 1976:56:489-91.

3 Rodenas JM, Delgado-Rodriguez M, Herranz MT, et al. Sun exposure, pigmentary traits, and risk of cutaneous malignant melanoma: a case-control study in a Mediterranean population. Cancer Causes Control 1996:7:275-83.

4 Evans RD, Kopf AW, Lew RA, et al. Risk factors for the development of malignant melanoma-I: Review of case-control studies. J Dermatol Surg Oncol 1988; 14:393-408.

5 Marrett LD, King WD, Walter SD, et al. Use of host factors to identify people at high risk for cutaneous malignant melanoma. Cmaj 1992; 147:445-53.

6 Elwood JM, Jopson J. Melanoma and sun exposure: an overview of published studies. Int J Cancer 1997;73:198-203

7 Vajdic CM, Kricker A, Giblin M, et al. Eye color and cutaneous nevi predict risk of ocular melanoma in Australia. Int J Cancer 2001;92:906-12.

8 Bataille V, Sasieni P, Cuzick J, et al. Risk of ocular melanoma in relation to cutaneous and iris naevi. Int J Cancer 1995;60:622-6.

9 Seddon JM, Gragoudas ES, Glynn RJ, et al. Host factors, UV radiation, and risk of uveal melanoma. A case-control study. Arch Ophthalmol 1990;108:1274-80.

10 van Hees CL, de Boer A, Jager MJ, et al. Are atypical nevi a risk factor for uveal melanoma? A case-control study. J Invest Dermatol 1994; 103:202-5.

11 Reese AG. Pigment freckles of the iris (benign melanomas): their significance in relation to malignant melanoma of the uvea. Am J Ophthalmol 1944;27:217-26.

12 Wilder HC. Relationship of pigment cell clusters in the iris to malignant melanoma of the uveal tract. Bull NY Acad Sci 1948;4:137-42.

13 Horn EP, Hartge P, Shields JA, et al. Sunlight and risk of uveal melanoma. J Natl Cancer Inst 1994:86:1476-8.

14 Michelson JB, Shields JA. Relationship of iris nevi to malignant melanoma of the uvea. Am J Ophthalmol 1977;83:694-6.

15 Tucker MA, Hartge P, Shields JA. Epidemiology of intraocular melanoma. Recent Results Cancer Res 1986;102:159-65.

16 Hale PN, Allen RA, Straatsma BR. Benign melanomas (nevi) of the choroid and ciliary body. Arch Ophthalmol 1965;74:532-8.

17 Ganley JP, Comstock GW. Benign nevi and malignant melanomas of the choroid. Am J Ophthalmol 1973;76:19-25.

18 Grulich AE, Bataille V, Swerdlow AJ, et al. Naevi and pigmentary characteristics as risk factors for melanoma in a high-risk population: a casecontrol study in New South Wales, Australia. Int J Cancer 1996;67:485-91. 